\title{
SCIENCE'S COMPASS
}

independently, perhaps by providing separate inputs to a common structure such as the hippocampus? The fMRI method cannot fully address this question. Lesion studies are required to determine which of these regions is actually necessary for new memories to be formed. And electrophysiological studies, with electrical or magnetic measures, are needed to achieve the temporal resolution necessary to determine if these regions are activated serially or concurrently. In short, the full benefits of functional neuroimaging data are gained when these are used to inspire and inform studies using other methodologies.

\section{References and Notes}

1. A. Baddeley, Human Memory: Theory and Practice (Erlbaum, Hillsdale, NJ, 1990).
2. L. R. Squire and S. M. Zola, Philos. Trans. R. Soc. Lon don Ser. B 352, 1663 (1997)

3. J. B. Brewer et al., Science 281, 1185 (1998); A. D. Wagner et al., ibid., p. 1188.

4. Reviewed in M. D. Rugg, in Electrophysiology of Mind M. D. Rugg and M. C. H. Coles, Eds. (Oxford Univ. Press, Oxford, 1995), pp. 132-170.

5. E. Tulving et al., Proc. Natl. Acad. Sci. U.S.A. 91, 2016 (1994); reviewed in P. C. Fletcher, C. D Frith, M. D. Rugg, Trends Neurosci. 20, 213 (1997)

6. C. E. Stern et al., Proc. Natl. Acad. Sci. U.S.A. 93,8660 (1996); J. D. E. Gabrieli et al., Science 276, 264 (1997).

PERSPECTIVES: CLIMATE CHANGE

\section{Just Add Water Vapor}

\section{David Rind}

$\mathbf{F}$ or those who believe that global warming will not be an issue in the next century, the behavior of water in the atmosphere is crucial. Global climate models show that increased warming will lead to greater evaporation from the ocean; with doubled carbon dioxide and more water vapor (an excellent greenhouse gas), the warming would be substantially more than $2^{\circ} \mathrm{C}$. Add to this the expected retreat of sea ice and snow cover, which allows more sunlight to be absorbed, and the warming reaches $3^{\circ} \mathrm{C}$ (more than half of the change since the last ice age). Yes, clouds may act to mitigate things, but they may also amplify the warming, as is the case in many climate models. Aerosols, such as sulfates released during fossil fuel burning, may slow the warming by reflecting sunlight away from Earth, but because they are somewhat easier to limit than $\mathrm{CO}_{2}$, we will probably clean up the air for health reasons and so exacerbate the warming.

As the lower tropospheric water vapor concentration would likely increase along with surface air temperatures, recent attention has focused on the upper troposphere, which is equally as important in terms of radiative transfer. Lindzen suggested that with increased convection and more vertical motion in the tropics, there would be increased subsidence bringing dry air down from higher levels and decreased moisture above about $6 \mathrm{~km}(1)$. Note how this deviates from model predictions (see figure, where upper tropospheric water vapor increases about $20 \%$ per degree Celsius of surface warming).

$\mathrm{I}$, as well as many others in this field believe that future reductions in high-altitude moisture are very unlikely; they would have to co-exist with low-altitude increases in an atmosphere that has many ways to mix moisture vertically. Furthermore, unless the current climate just happens to have the maximum moisture aloft,

The author is at NASA/Goddard Institute for Space Studies, New York, NY 10025, USA. E-mail: drind@giss.nasa.gov the theory also implies that colder climates should be wetter at high altitudes; with less evaporation and convection, where would the moisture come from, and how would it get up there? Such arguments aside, it would nice to be able to test either expectation or, perhaps more importantly, to determine whether climate models produce the proper magnitude of the water vapor feedback. Unfortunately, we do not

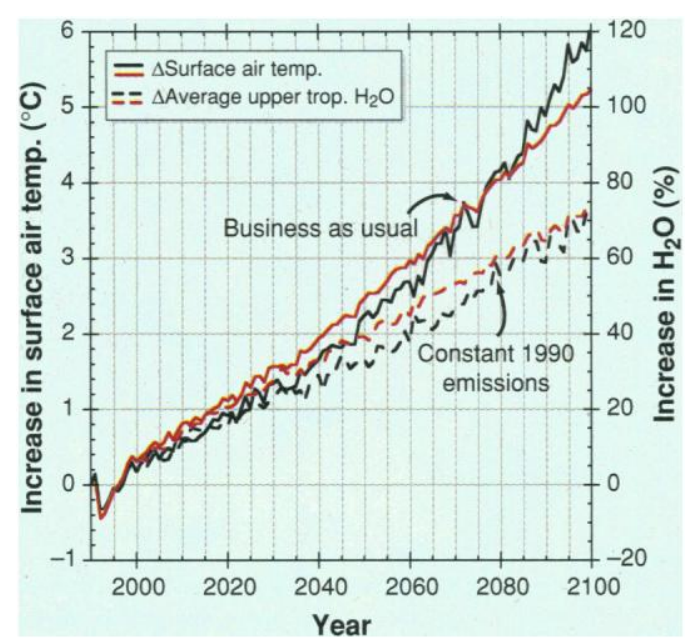

Wetter weather. Surface air temperature and percentage change in specific humidity in the upper troposphere (trop.) from the GISS model for two scenarios of trace gas emissions: business as usual (solid lines) and the (climatically ineffective) constant 1990 emissions (dashed lines). No aerosol increases are used, and, given the relatively high model sensitivity $\left(1^{\circ} \mathrm{C} \mathrm{W}^{-1} \mathrm{~m}^{-2}\right)$, this represents almost a worst case scenario. For reduced warming, the change in upper tropospheric humidity would be proportionately less, and thus the accuracy needed to observe it would need to be that much greater.

have the observations in place to be able to do so, and it is not clear when we will.

Historically, water vapor values above the surface have been measured by sensors on radiosondes. As is well known, these cannot be used to assess trends: They are geographically restricted, being found mostly over land in the Northern Hemisphere, and worse still, different sensors give differ- ent absolute water vapor values. Poorer instruments respond too slowly and overestimate water vapor amounts at high levels. As these deficiencies become known, and countries (including the United States) shift to better sensors, it may well appear that the upper troposphere is drying simply because of the better instrumentation (2).

Satellites, with global data collection, ideally could provide an answer for the recent past except that they have their own problems. Downward-looking instruments cannot see with good vertical resolution; results are generated for 300-mbar-thick layers (that is, layers defined on a pressure scale), which are too coarse to properly calculate radiative forcing changes. Limb-scanning instruments have large horizontal footprints, which are tricky to interpret for a constituent so heterogeneous in space and time. Furthermore, they were designed for stratospheric monitoring and tend to have large error bars for data collection in the upper troposphere. Clouds also present problems, lowering the accuracy even for microwave instruments. New sensors becoming available as part of NASA's EOS research program, as well as for European and Japanese missions (and ultimately to be used on operational satellites), will have better radiometers, but it is yet to be proven that they can overcome these difficulties. And, with cutbacks in the EOS program by the U.S. Congress and a certain disinterest on NASA's part in acting as a monitoring agency, current plans do not envision most of them remaining aloft long enough for trends to be determined anyway.

Stratospheric water vapor increases could also amplify the greenhouse effect and, with less variability and cloud cover interference, should be easier to measure (doubled stratospheric water vapor provides radiative forcing of about $1 / 7$ that of doubled atmospheric $\mathrm{CO}_{2}$ ). There are indications that it might already be increasing (3). Unfortunately, here too measurement 
problems exist: In situ sensors do not agree, and satellite instruments lack sufficient absolute agreement to provide consistent trends (4). Even if stratospheric trends are more determinable, it will not necessarily imply anything about the upper troposphere; if tropopause temperatures increase, more water vapor could get into the stratosphere without implying there is more water vapor below.

So given these difficulties, researchers are attacking pieces of the problem. Efforts have been made to assess whether vertically integrated water vapor changes have been occurring; the general conclusion is yes, but it is not unanimous (5). On shorter time scales, drying does occur in the upper troposphere immediately after convective events, but wetter conditions soon follow, presumably as the associated cloud cover dissipates (6). Considering annual cycle variations, water vapor and the global atmospheric greenhouse effect do increase with surface temperature, especially in the tropics (7). Nevertheless, it is still uncertain how such effects relate to what happens as climate itself changes.

Where does this leave us with respect to global climate change estimates? So far, there has been no evidence to indicate that a strong negative water vapor feedback in the upper troposphere will in fact arise as climate warms. However, without our being able to observe upper tropospheric and stratospheric water vapor with sufficient accuracy over a long enough time period to see ongoing trends, some uncertainty will remain in this most important of climate sensitivity feedbacks.

\section{References}

1. R. S. Lindzen, Bull. Am. Meteorol. Soc. 71, 288 (1990).

2. W. P. Elliott and D. J. Gaffen, ibid. 72, 1507 (1991).

3. S. J. Oltmans and D. J. Hofmann, Nature 374, 146 (1995); S. Evans, R. Toumi, J. E. Harries, M. Chipperfield, J. M. Russell III, J. Geophys. Res. 103, 8715 (1998)

4. M. P. McComrick and E.W. Chiou, paper presented at the American Geophysical Union Spring Meeting, Boston, MA, 26 to 29 May 1998; H. K. Rosenlof et al., paper presented at the American Geophysical Union Spring Meeting, Boston, MA, 26 to 29 May, 1998.

5. IPCC, in Climate Change 1995,.J. T. Houghton et al, Eds. (Cambridge Univ. Press, Cambridge, 1995); S. R. Schroeder, and J. P. McGuirk, Geophys. Res. Lett. 25, 1301 (1998).

6. X. Liao and D. Rind, J. Geophys. Res. 102, 19543 (1997).

7. A. K. Inamdar and V. Ramanathan, in preparation.

\section{Electroweak Reconciliation}

\section{Michael E. Peskin}

$\mathrm{H}$ igh energy physicists view themselves as intrepid explorers, searching for new concepts of space and time. So it is sometimes difficult for them to accept that the theories in their textbooks actually work well. Over the past few years, however, experiments have tested the structure of the weak interaction - the basic force that gives rise to radioactive decay-and, with remarkable precision, the results have converged on the standard textbook model. The experiments confirm the elementary couplings of this interaction to the basic constituents of matter, the leptons (such as the electron) and the quarks (from which the proton and other nuclear particles are made). The observed large mass of the heaviest quark, the top quark, plays a key role in this reconciliation. The results close a chapter in the history of particle physics and present some interesting clues to the content of the next chapter.

The heavy particles $W^{ \pm}$and $Z^{0}$, which mediate the weak interactions, were discovered in 1982 at CERN (1). The masses of these particles are 80 and $91 \mathrm{GeV}$, respectively (for comparison, the proton mass is about $1 \mathrm{GeV}$ ). In 1989, the inauguration of two new machines, the Stanford Linear Collider (SLC) and the Large Electron-Positron (LEP) collider at CERN, allowed precision studies of the properties of the $Z^{0}$. An important property of the weak interaction is that it violates parity (as reflected in the radioactive decay of nuclei, which produces electrons and neutrinos

The author is at the Stanford Linear Accelerator Center, Stanford University, Stanford, CA 94309, USA. E-mail: mpeskin@slac.stanford.edu with left-handed, as opposed to right-handed, spin). This spin asymmetry leads to distinctive effects in $Z^{0}$ decays.

The coupling of the $Z^{0}$ to each species of quark and lepton is predicted by the weak-interaction theory of Glashow, Salam, and Weinberg (1). This theory contains four fundamental particles - two electrically charged and two neutral-which are responsible for the weak and the electromagnetic (collectively, "electroweak") interactions. At the basic, symmetrical level of the theory, all four particles are massless. To give mass to these particles, it is necessary to assume an additional field, existing throughout space. This is known as the "Higgs boson field," and it gives mass to the weak-interaction bosons according to a specific pattern. The charged particles acquire mass and can be identified with the $W^{ \pm}$. The two neutral particles mix with one another by a small amount, and one of these particles remains massless. This massless state is precisely the photon. The remaining state is the $Z^{0}$, which obtains a mass slightly larger than that of the $W^{ \pm}$. The mixing angle is a single parameter that determines the coupling strengths and spin asymmetries of all of the individual quark and lepton species. The predicted spin asymmetries differ greatly, from about $14 \%$ for the electron to $94 \%$ for the $b$ or bottom quark. Through systematic measurements, at LEP and SLC, of the angular distributions and decay patterns of particles produced from the $Z^{0}(2)$ and through an experiment at SLC that directly measures the rate of $Z^{0}$ production separately from left- and righthanded electrons (3), these various values for the spin asymmetries are confirmed experimentally

The most accurate way to determine the mixing parameter is to combine a precise absolute measurement of the $Z^{0}$ mass with two quantities that are already known to part-per-million accuracy, the rate of muon beta decay and the electromagnetic finestructure constant. In the past 2 years, the LEP collider at CERN has been calibrated to five-decimal-place accuracy (a level at which influences of the tides, the water level in Lake Geneva, and local railroad operation must be identified and subtracted) to give a very precise determination of the $Z^{0}$ resonance position (4). The decay rate and spin asymmetry measurements for the various species give additional measurements of the mixing to four decimal places, which provide detailed tests of the model.

The accuracy of these experiments is such that they cannot be compared without taking into account the higher order quantum mechanical corrections. Exotic quantum processes involving the weak interactions play an equal role with high-order quantum electrodynamics processes in the computation of these corrections. Both types of effects influence the predictions for weak-interaction rates and asymmetries at the $1 \%$ level of accuracy. Thus, the detailed comparison of different experiments that measure the mixing parameter can determine whether these quantum processes are actually present to the extent predicted by the theory. Although the full structure of the corrections is rather complicated, the most important effects come from the "vacuum polarization" process, in which a $Z^{0}$ converts for a short time, by a quantum fluctuation, into a pair of electrons, quarks, or $W$ bosons. In principle, the $Z^{0}$ could also fluctuate to a pair of heavier particles, indeed, to any particle that couples to the weak in- 